\title{
INTEGRATED LAND-USE AND TRANSPORTATION MODELLING IN DEVELOPING COUNTRIES: USING OPENTRIPPLANNER TO DETERMINE LOWEST-COST COMMUTE TRIPS
}

\author{
LOUIS WALDECK \& QUINTIN VAN HEERDEN \\ Spatial Planning and Systems, Built Environment, Council for Scientific and Industrial Research, \\ Pretoria, South Africa
}

\begin{abstract}
Large cities are amongst the most complex production systems ever built. In this chapter the role of Land Use Transportation Interaction (LUTI) models in supporting the equally complex urban planning processes that shape our cities is being explored. Specific reference is made to developing countries where poverty, income disparities, and mounting demands for basic services pose even greater challenges to urban planners. The paper refers to the urban simulation model that was developed for South Africa by adapting UrbanSim and OpenTripPlanner and how this combination allowed practitioners to reduce the onerous data preparation and simulation times that have become characteristic of leading micro-simulation LUTI models. In the chapter the case for using the monetary cost of commuting as an acceptable proxy for the generalised cost because low-income households in developing countries tend to be more sensitive to the cost of a trip than the duration is demonstrated. The results from two case studies in different South African cities are presented and the combined model is validated by simulating a ten-year period between the last two population censuses.
\end{abstract}

Keywords: transportation modelling, urban modelling, integrated land-use and transportation, developing countries, UrbanSim, OpenTripPlanner.

\section{INTRODUCTION}

In the developing world, mobility challenges and the role of government in the provision of infrastructure are significantly different from those in the developed world. Confronted by extreme poverty, income disparities and mounting demands for basic services such as clean water, sanitation and health care, urban planners in developing countries face daunting challenges, Cervero [1]. In Sub Sahara Africa, 62 percent of urban households reside in shantytowns and 64 percent of those with formal jobs lived on less than US\$1.25 per day in 2010, United Nations [2]. In South Africa, 20 percent of households lived in informal settlements (shantytowns) and a further 11 percent in informal backyard dwellings in 2011 according to Statistics South Africa [3]. The median personal income was about US\$14 per day based on average exchange rate of US\$1 = ZAR6.9 in 2011.

Another challenge to the Government of South Africa is striking a balance between investment in 'economic infrastructure' such as transportation and energy supply systems and 'social infrastructure and services' including water, sanitation, health and a variety of grants/subsidies aimed at alleviating the plight of the poor without expecting a monetary return on the investment. Public transportation has attracted massive investments since the promulgation of the National Land Transportation Act of 2009. Like in most cities around the world the new public transportation services are subsidised, but probably more so as a service to the poor and this chapter will endeavour to highlight the significant benefits of 
subsidisation. The jury is however out on whether target densities will be reached within the designated transportation corridors to achieve the ridership levels required to contain subsidies.

Since urban decision making processes can be adversarial due to differences in opinion about the policy scenarios that should be adapted to build a more sustainable city, it is no wonder that a myriad of planning support models have over the years made their appearance to assist decision makers by simulating the likely outcome of policy scenarios. The relative success of a policy scenario in achieving one or more objectives is then evaluated by comparing a number of predefined indicators. For example, in a public transport corridor densification scenario, the success of public transport and higher density zoning could be measured by comparing a simple indicator such as the resultant population density within the confines of the corridor, with and without public transport.

The value of these models is not in predicting the future, which it obviously cannot do, but in providing participants in the planning process with a better understanding of cause and effect between different components of the urban system, which ranks among the most complex production systems ever built and in discovering common ground between different departments and interest groups that could lead to compromise. We thus argued that such tools are even more relevant in developing countries due to the additional challenges outlined at the outset.

The class of models suitable for this type of work is commonly referred to as Land Use Transportation Interaction (LUTI) models. The literature abounds with a daunting number of LUTI models and many papers have been written on the topic of comparing models in terms of criteria such comprehensiveness, theoretical foundations, data requirements, etc. In one of the earlier papers Wegener [4] concluded that "The most promising technique for activitybased land use and transport modelling is micro simulation which makes it possible to reproduce the complex spatial behaviour of individuals on a one-to-one basis". Nearly a decade later Wegener [5] concluded that the trend towards greater disaggregation has gone mainstream, activity-based travel models have become the state-of-the-art and agent-based land-use models are proliferating. Simulation runs for leading micro-simulation models are regrettably now measured in terms of days or weeks, not hours.

In South Africa the Council for Scientific and Industrial Research (CSIR) developed an Urban Simulation Model that has been used to simulate spatial growth patterns 30 years into the future, to better understand the future demand for infrastructure, facilities and services including water, sanitation, electricity and transportation. The model was initially based on UrbanSim and MATSim but is currently based on adapted versions of UrbanSim and OpenTripPlanner (OTP). The change from MATSim to OTP was partly motivated by the need to achieve a significant improvement in computational efficiency and partly to build a model that would be more representative of a developing country like South Africa.

In this chapter the rationale for adapting OTP is presented and applied as a substitute for MATSim, the nature of the interaction between UrbanSim and OTP and discuss the advantages and limitations of the approach. The results of successfully applying the Urban Simulation Model in the Cities of Tshwane and Nelson Mandela Bay is being presented. To start off with, the scope of a typical LUTI model is explored before providing a brief overview of UrbanSim.

\section{SCOPE OF LAND USE TRANSPORTATION INTERACTION MODELS}

In a comparative study of twenty contemporary models Wegener [4] identified the eight important urban subsystems in Fig. 1, of which UrbanSim addresses 7. Other models addressed a minimum of two and maximum of eight subsystems. 


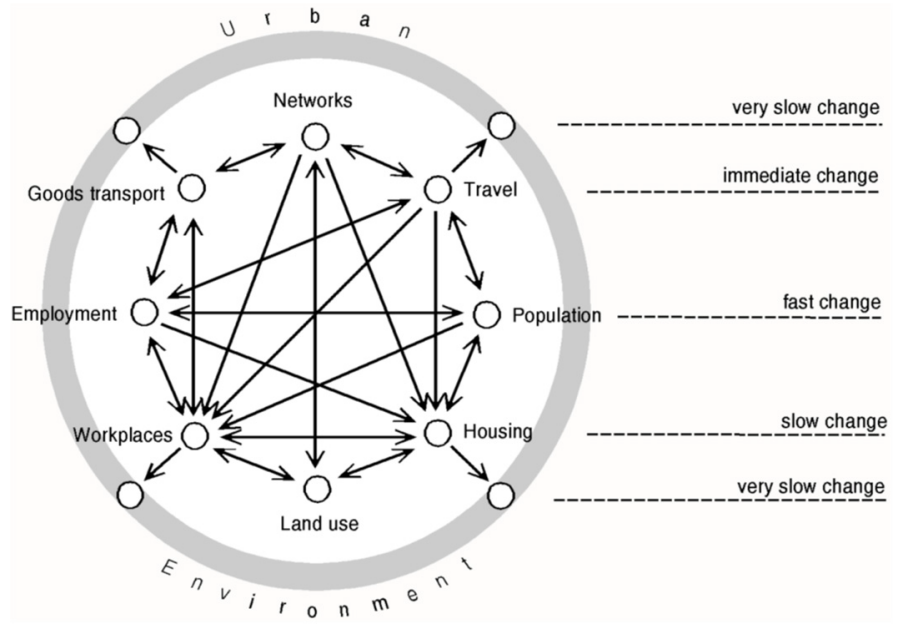

Figure 1: Web of urban processes (from Waddell [6], after Wegener [7]).

From the perspective of the actors involved in various subsystems one could summarise the above diagram as follows: Households decide where they live, where they work and how they commute between the two. Employment opportunities are created by the location decisions of firms. Developers provide housing and floor space for conducting business after calculating the expected return on investment of development proposals and constructing proposals that maximise their profit. Government provides transportation networks and regulates physical development by virtue of the rights associated with each parcel of land within a policy environment.

The spatial distribution of residential, business and industrial land uses in particular determines the location of human activities such as living, working and shopping. The demand for transportation is a direct result of the separation of these activities. The supply of transportation in turn determines how accessible places of interest are, which may in turn influence the choice of location of households and thereby the demand for transportation to complete the 'land-use transport feedback cycle'.

The literature abounds with a daunting number of available LUTI models. A decade ago already Wegener [4] pointed out that there were significant variations among models with respect to comprehensiveness, model structure, theoretical foundations, modelling techniques, dynamics, data requirements and calibration and validation. Based on his more recent research findings from the EU-funded SustainCity programme, Wegener [5] found that the primary difference between models can be reduced to: (1) whether a model represents the urban system as a dynamically changing system or one in equilibrium, and; (2) the degree of disaggregation allowed by a model to capture the heterogeneity in human behaviour resulting from individualisation in modern societies. The latter is particularly important due to various forms of inequality that are characteristic of the South African context.

In another review by Simmonds et al. [8] UrbanSim was considered as best practice (with two other LUT models: DELTA and IRPUD) in terms of quasi-dynamic models (there are no fully dynamic models available yet) by virtue of subsystem-specific sub models.

From the foregoing it can be concluded that UrbanSim finds itself in an elite group of state of the art models. In the next section we provide a brief overview of UrbanSim before returning to OpenTripPlanner. 


\section{OVERVIEW OF URBANSIM}

UrbanSim recognises the property market as the central construct of the urban system and accounts for the current stock of real estate (and its spatial distribution) as well as the supply and demand of new stock. In Fig. 2, households and businesses represent the consumers of residential and non-residential stock respectively. The choice of location by these agents is accounted for by the occupation of space in a building.

Developer agents, representing the supply side, use occupancy rates as a feedback signal to determine the rate at which new stock can be built and sold. Supply and demand are mediated by price, another feedback signal which influences the return on investment calculation done by developers.

Governments set policies that either regulate the use of land or influence development through pricing policies such as developer contribution fees. Governments also build infrastructure and provide social facilities such as libraries, clinics, community halls, parks, sports field and fire stations.

Transportation infrastructure and the quality of social facilities influence the attractiveness of locations for different consumers.

Households have particular characteristics that influence their preferences for housing of different types at different locations. Similarly, businesses have preferences that vary by industry and number of employees for alternative building types and locations. Two models are prominent in modelling these choices, the Household Location Choice Model and the Employment Location Choice Model. Both are discrete choice models based on the pioneering work of McFadden on Random Utility Maximization Theory (McFadden [10] and McFadden [11]). This approach determines the probability of a choice from a set of available alternatives based on the characteristics of the chooser and the attributes of the alternatives, depending on the relative utility that an alternative offers the chooser.

The systematic component of a utility (as opposed to the random component) is expressed as a linear combination of estimable coefficients multiplied by independent alternativespecific variables that may be interacted with the characteristics of the household making the choice. The coefficients are estimated using maximum likelihood methods built into UrbanSim. For choice models the estimation can be based on the stated or observed behaviour of agents. All research by the authors so far has been based on observed behaviour derived from sources such as census and household travel surveys.

Examples of alternative-specific variables often used in utility calculations include the distance, travel time and generalised cost of commuting. The term generalised cost refers to

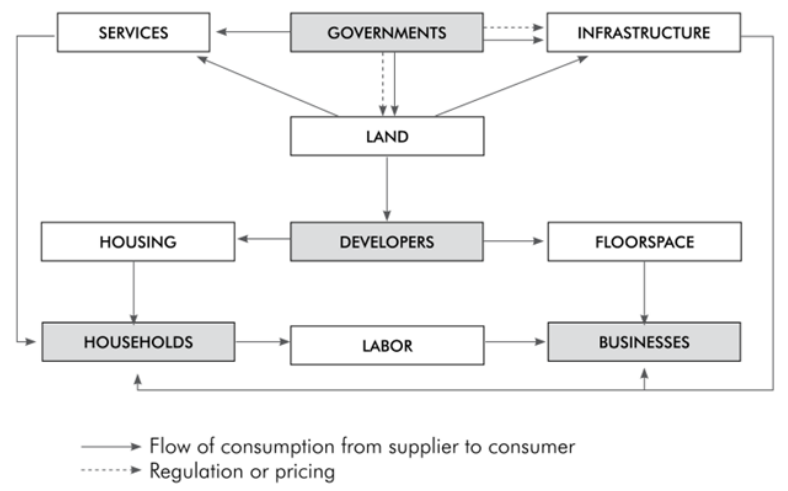

Figure 2: Interaction of agents with urban markets (from Waddell [9]). 
the sum of the monetary cost and the value of time spent undertaking the trip, which varies according to the traveller's income and the purpose of the trip.

The choice of variables is more of an art than a science. The process usually starts by considering some variables derived from urban economic theory. Guided by various indicators which estimate the relative importance and statistical significance of each variable, variables are added or deleted to maximise the overall explanatory power of the model. In South Africa the process presented a few more challenges, such as a negative correlation between low-income households and proximity to work opportunities. Whereas this would defy urban economic theory in a developed country, it is still a reality in South Africa due to the politically motivated spatial planning policies of the past.

It is unable to describe all the adaptations that were made to both UrbanSim and OpenTripPlanner in one chapter. The focus will thus only be on OTP and UrbanSim will be dealt with in future publications.

\section{RATIONALE FOR USING OTP AS SUBSTITUTE FOR MATSIM}

The investigation into using OTP as a substitute for MATSim was prompted by the research of Nicolai and Nagel [12] to determine if measures of accessibility based on pairs of locations could be replaced by measures based on attributes of the location itself, since this would significantly improve computational performance. As such, the study by Nicolai and Nagel was undertaken with the objective of improving the interaction between UrbanSim and MATSim rather than finding ways of avoiding it. As long as travel times or generalised costs are used in the calculation of accessibilities, such calculations will have to be based on the congested state of transportation networks.

But what if the monetary cost of a commute trip can be used as acceptable proxy for generalised costs in South Africa, and perhaps other developing countries? In that case, cost and distance-based measures of accessibility would no longer depend on the congested state of networks and since the monetary costs and network-distances can be obtained from OTP, no need for interaction with MATSim, at least not for the purpose simulating urban growth, exists.

The remainder of this section explains how this possibility was investigated. Firstly, the underlying causes of long simulation runs were examined, then a high resolution accessibility measures as a means of improving computational efficiency is introduced and finally the argument, based on the household income distribution of South Africa that a model based on monetary costs could be a more representative model of reality in South Africa is developed. From this, the potential for other developing countries is deduced.

\subsection{Interaction between UrbanSim and MATSim}

Travel times and generalised costs are usually obtained from a series of interactions between UrbanSim and a transportation model that represents the congested state of the transportation network, such as EMME, VISUM or MATSim. Interaction is achieved by way of an exchange of data at the start of every UrbanSim simulation year as illustrated in Fig. 3. The effect of this simple action is unfortunately to increase the total execution time for a 30 -year simulation period by many hours or even days depending on the size of the study area. Although some progress has been made with parallelising UrbanSim (Awaludin and Chen [13]) and MATSim (Raney and Nagel [14]) it is unlikely that the coupled system will benefit in the same way from advances in parallel computing because one model has to finish before the other can start with the next year. It is therefore common for modellers to introduce austerity measures such as restricting interaction to 5 or 10 year intervals. 


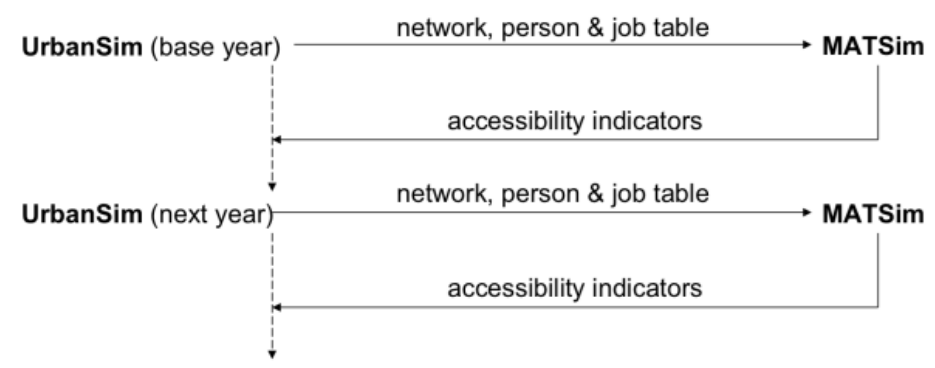

Figure 3: Sequence of interaction between UrbanSim and MATSim (from Nicolai and Nagel [12]).

\subsection{High resolution accessibility measures}

In an attempt to resolve some of the above constraints, Nicolai and Nagel [12] investigated the notion of high resolution accessibility to determine if measures of accessibility based on pairs of locations could be replaced by measures based on attributes of the location itself. The study was prompted by the observation that many of the variables used in specifications of the Household Location Choice Model all over the world appeared to be simple accessibility measures anyway, for example distance to workplace, jobs within 30 min drive time, etc. This not only agrees with the experience of the authors and that of other papers from the EU-funded Sustain City project, for example Zöllig Renner et al. [15] but appears to also be a fair representation of household decision-making processes. Few, if any, households consult transportation models when considering the location of a new house. For road trips they probably assume that most roads are congested during peak times and use distance as proxy for comparing the expected commute time from the new location to their current commute time.

Hansen [16] defines accessibility as the potential of opportunities for interaction. If locations are otherwise equal, a location with easier access to activities like work, leisure or shopping is more attractive than locations with less access and have greater potential for residential development. Moeckel [17] confirms that this approach is also true for businesses.

In a study, Nicolai and Nagel defined the accessibility of location $i$ as $\log$ sum of generalised $\operatorname{cost} C_{i j}$ over all locations $j$ :

$$
A_{i}:=\ln \sum_{j} e^{-\beta C_{i j}}
$$

In high-resolution accessibility calculations, there are two resolutions to consider: one that determines for how many origins $i$ the accessibility is to be computed and another that determines to what level the destinations $j$ need to be resolved.

The number of origins was made variable in the form of a grid measuring 250, 500, 1000, $2500,5000,7500$ and 10000 meter per side. The number of destinations is taken as a $10 \%$ sample of all job locations (more precisely the network nodes closest to the buildings with these jobs).

Nicolai and Nagel applies an existing UrbanSim/MATSim model of the Puget Sound Regional Council (which includes Seattle) to investigate the feasibility of calculating high resolution accessibilities for each of the seven origin square sizes. A generalised costs $C_{i j}$ from the first MATSim year is used as representative of the congested state of the network. 
The results demonstrate that it is computationally feasible to, for every origin; calculate a weighted sum over all possible destinations separately, rather than aggregating them into zones. This removes all zonal artefacts from the destination side of the computation, such as job opportunities in neighbouring zones being easier to reach than opportunities within the zone itself. It is also concluded that the spatial resolution of the origins had a strong impact, with resolutions smaller than $1000 \mathrm{~m} \times 1000 \mathrm{~m}$ producing diminishing returns.

\subsubsection{Origins and destinations}

Regarding the spatial resolution of origins and destinations, UrbanSim applies three geographies: user-defined grid, land parcels or user-defined zones. The research focus of this chapter is, thus far, based on something between parcels and zones. It commences with individual property boundaries but later on combines adjacent parcels in a street block that are indistinguishable in terms of a number of attributes into a single parcel and represent all the (similar) buildings on the individual parcels as one pseudo building per street block. This reduces the number of parcels and buildings by a factor of roughly 20 with distinct advantages for GIS intensive data preparation and some reduction of UrbanSim execution times, without affecting the explanatory power of the model. Households residing in the same street block are not combined and dissimilar properties such as residential and commercial are likewise not combined.

With OTP it is perfectly feasible to determine an accessibility indicator for every street block even if this requires the lowest cost route over all modes of transport to be calculated from every street block to every other street block. This is made possible by the fact that OTP 'remembers' the result of previously considered routes to avoid unnecessary recalculation.

Mindful of the conclusion reached by Nicolai and Nagel [12] that little is gained by using origins smaller than about $1 \mathrm{~km}^{2}$, street blocks that are typically 20 to 50 times smaller, is considered to be wasteful. Another factor that influences the choice of resolution is that the notion of a street block is only useful for areas that are already developed or in the process of being established. In outlying areas the smallest property boundary in the base year of a simulation could be as large as a farm. Using such parcels as unit of analysis in longitudinal studies of urban growth could lead to misleading results due the Modifiable Areal Unit Problem (MAUP). To avoid such complications, it was decided to use 'modified small areas' or 'modified sub places' both as origins and destinations for OTP and as unit of analysis for UrbanSim. In the rest of the chapter these will simply be referred to as 'zones'. 'Sub places' and 'small areas' are two of the units of analysis at which census information is released by Statistics South Africa (StatsSA). The authors modify these areas by retaining the smaller ones in built-up areas, sometimes combining very small ones and subdividing the larger ones (and where necessary the corresponding parcels) in order to obtain a more even size distribution. In the case of Nelson Mandela Bay the zones on modified small places with a median area of $0.9 \mathrm{~km}^{2}$ and a maximum of $2.3 \mathrm{~km}^{2}$ are being used.

Note that while the number and size of zones affect the number of lowest cost routes to be found by OTP, it does not affect the street-block resolution of UrbanSim simulations, merely the spatial resolution to which the results are aggregated afterwards for analysis and interpretation purposes.

\subsubsection{Changing location of simulated jobs}

One of the initial attractions to investigate the notion of accessibility measures based on the monetary cost of commuting is that such measures no longer depends on the congested state of networks and only needs recalculation when there is a significant change to the road or transit network. 
If the accessibility measures are passed back to UrbanSim in the same way as contemplated in Fig. 3, it is only be valid for one year because the location of jobs keep changing from one simulation year to the next. To avoid this it was decided to store the OTP results of the lowest-cost routing for different modes of transport per Origin-Destination (OD) pair. This allows the use standard UrbanSim functionality for defining variables to calculate measures of accessibility based on the correct job locations for each simulation year. It also opens up numerous other possibilities to customise accessibility to jobs in different sectors of the economy, for households of different income groups, etc.

This solution opposes the efforts of Nicolai and Nagel [12] to get rid of storing OD pairs, but is well worth it if it avoids repeatedly running OTP or MATSim. For example, in Nelson Mandela Bay with 2672 zones, the OD matrix has 7.14 million rows and 6 columns, taking up $512 \mathrm{MB}$ of storage. All OTP related processing take about 30 minutes on an ordinary laptop computer and as mentioned before it only has to be repeated when a different scenario requires the network to change.

If the OD matrix becomes a constraint for large study areas, the size could be cut in half by changing it to a symmetrical matrix on account of the fact that the cost of a trip from A to B will only differ marginally from the trip from B to A where one-way streets are involved. For the focus of this research, it does not pose a limitation.

\subsection{Accessibilities based on the monetary cost of commuting}

Having explain how measures of accessibility such as the log sum defined in eqn (1) may be applied as an UrbanSim variable and used in the Household Location Choice Model to influence the location choices of households, it is now possible to move considering whether accessibility measures based on the generalised cost of commuting can be replaced by measures based on monetary costs in South Africa and perhaps elsewhere in the developing world. This premise does not imply that the same approach is to be followed in the developed world.

The difference between using monetary costs and generalised costs is that the latter depends on the congested state of the network and introduces another complication in the form of the value of time, which is not easy quantifiable, even in the developed world. One of the difficulties with the value of time is the difference between the stated and observed behaviour of households. If high-income households residing in security areas such as golf and eco estates on the periphery of South African cities, are surveyed, they will put a high value on time. The conclusion may thus be drawn that location choice adds to daily travel time that location choices travel time is of less importance and less valued. The answer is often that there are other factors which are even more important than travel time, such as safety and security considerations, which are often quoted as a reason for the popularity of such estates.

Apart from the value of time, the argument for using measures of accessibility based on monetary cost in South Africa is based mainly on an income distribution which has a large proportion of poor households. It is well known that low-income households have to spend proportionally more on transportation than higher income households and it is concluded from the research that the lowest income groups (typically found in informal settlements or backyard dwellings) follow scarce job opportunities to minimize expenditure on transportation, often by opting for non-motorised modes (especially walking) of transport. 


\subsubsection{Household income}

For the purpose of determining what percentage of households could be considered to belong to 'lower income groups' various studies dealing with the literal and other more nuanced definitions of the 'middle class' were consulted, for example Visagie [18] and Visagie and Posel [19]. The authors of this chapter eventually decided to define the 'lower income group' as all households with income less than the lower limit of the more nuanced 'middle class' shown as 'Relatively Affluent Middle' in Fig. 4 below. This point of departure is based on the National Income Dynamics Study (NIDS) of 2008. Although the actual breakpoints differ slightly from the Census of 2011 , the overall conclusion is that about $65 \%$ of households are classified in the category of 'low income'.

Since the ratio of household expenditure on a given mode of transportation to household income increases with decreasing income, it would be expected that the location choices of poorer households to be more sensitive to trip cost than travel time. This is one reason why accessibility measures based on monetary costs are a more representative model for SA than one based on travel times and generalised cost.

It, however, should not be confused with the determinants of modal choice and location preference. Due to this statement, it is useful to explore the results of the National Household Travel Survey of 2013 [20].

\subsubsection{National Household Travel Survey}

The figures quoted below differ from the published Statistical Release [20] because they are based on an analysis of a subset of data representing the six largest metropolitan cities that the Urban Simulation Model would typically be applied to.

In application of research related to travel the following findings are applicable: In the bottom four quintiles $5 \%$ of workers walk to work; another $80 \%$ use public transport (i.e. minibus taxi, bus, and train) and a mere $15 \%$ private transport as driver or passenger. In the top quintile the corresponding figures are $2 \%$ of workers walk, $35 \%$ use public transport

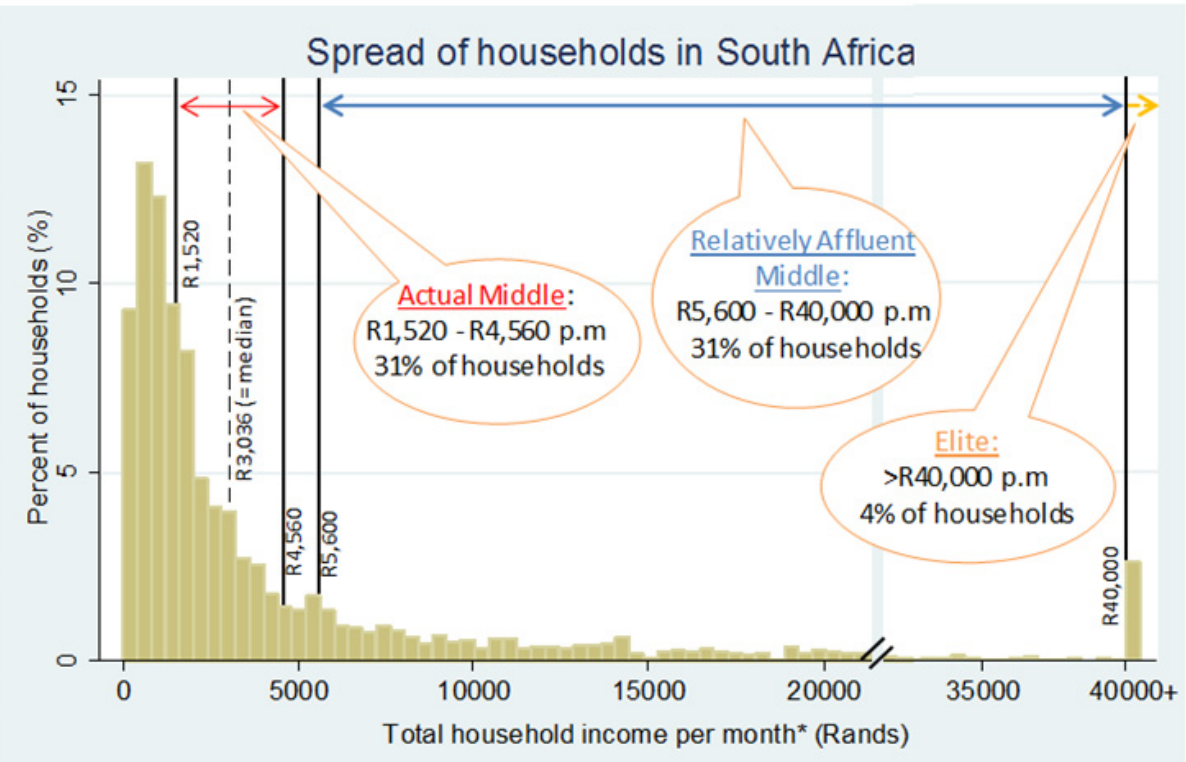

Figure 4: Household income distribution (from Visagie [18]). 
and the remaining $63 \%$ private transport. Regarding the factors that influence the preferred choice of a particular mode the following finding applies: In the bottom two quintiles more workers cited travel cost than travel time, roughly equal numbers in the third quintile and more workers cited travel time than travel cost in the top two quintiles. Also of interest is that factors such as flexibility of time and destination increase from $5 \%$ in the bottom four quintiles to $15 \%$ in the upper quintile.

The interpretation of the abovementioned results is as follows: For $60 \%$ to $80 \%$ of households, cost is critical. Based on the observation that the percentage of workers that walk all the way to work peaks in the bottom three quintiles it is unlikely to be motivated by healthier living considerations, more likely a survival or household budget balancing mechanism. Even where travel time is cited as more important than travel cost it is probably expressing a preference for a faster minibus taxi service. Of the $80 \%$ of workers that rely on public transportation $51 \%$ use minibus taxis, nearly double the number of workers that rely on train $(15 \%)$ and bus $(13 \%)$ services. Due to government subsidies, the fare structures for public transport are such that Metrorail fares are the cheapest, bus fares approximately double that, and minibus taxis, which are regarded as public transport but not subsidised, approximately double the bus fares. This creates an environment where commuters from the bottom two quintiles choose Metrorail where available, then bus and then minibus taxis. For commuters from households in the next two quintiles this may change to Metrorail when and where convenient, then a choice between a slower bus service and a faster minibus taxi or BRT service depending on whether travel time or travel cost are most important to the commuter.

For $20 \%$ of households in the upper quintile, $63 \%$ of workers use private transport. These households have the means to make lifestyle choices and some prefer to live in security, golf or eco estates that are not centrally located. It may be expected that travel time would be important to these households but as pointed out earlier this may be overridden by safety and security considerations or there may simply be no public transport available in such areas.

In conclusion, the research shows that a model based on monetary costs offers an acceptable if not better representation/reflection of the reality facing $80 \%$ of households in South Africa. Does this approach sacrifice anything in terms of how the remaining $20 \%$ of households are accommodated in terms of the model as developed? To answer this question it must be pointed out that the implementation of UrbanSim deals with five income groups in different sub models of the Household Location Choice Model, allowing completely different variables to be used as predictors of behaviour for each income group. Only two variables, travel time and generalised cost are not available as a result of using OTP. It has been pointed out that it is no trivial matter to quantify the value of time. Even when travel times were available in the past, the research rarely found travel times to be more significant predictors of behaviours than distance-based variables. This is in line with the finding from at least one of the case studies of the SustainCity project, in which Zöllig Renner et al. [15] concluded that the distance to workplace to be the $2^{\text {nd }}$ most important variable with accessibility measures based on generalised cost $4^{\text {th }}$ and $5^{\text {th }}$ most important. Also of interest is that affordability of rent or bond payment featured as the $3^{\text {rd }}$ most important variable, which correlates with the situation in South Africa.

\section{MODELLING TRIP COST USING OTP}

Having argued that utilities for household location choices can be based on accessibilities calculated from distance and monetary cost variables, the line of reasoning in this chapter, will focus on how to use OTP for such calculations. 


\subsection{Software}

The investigation for the focus of this research commences by considering various software solutions to compute the lowest cost route between a potentially large number of origins and destinations. The routing algorithm was required to consider all modes of transport, including distance limited walking and cycling, private vehicles, unscheduled passenger vehicles and mass transit (bus and rail), as well as possible transfers between any of these modes.

After considering various alternatives, including ArcGIS Network Analyst, NetworkX and OpenTripPlanner it was concluded to select the OpenTripPlanner (OTP). The decision was based primarily on the observation that OTP is purpose-made for routing, whereas ArcGIS and NetworkX are more general purpose tools that would require greater customisation than OTP to achieve the objective stated above. OTP Analyst already makes the transit system model and optimization logic originally developed for point-to-point searches available for one-to-many and many-to-many queries in large batches.

Using OTP for the purpose envisaged in this section involves preparing XML configuration files and then running the JAVA classes for OTP Graph Builder and OTP Batch Processor. The configuration files are required to set options and point the software to input and output folders and other simulation specific variables such as the maximum walking distance to a public transport stop. The Graph Builder converts an OSM file for the study area and a collection of General Transit Feed Specification (GTFS) [21] files into a routable graph object required by the Batch Processor to determine the lowest cost route between any number of origins and destinations. The origins and destinations could be provided as GIS point or polygon features or as coordinates in a text file.

\subsection{The General Transit Feed Specification}

The General Transit Feed Specification (GTFS) is a format developed by Google to publish spatial and temporal information of public transportation schedules. When an agency publishes its schedules in this format it becomes a GTFS feed. The schema allows application developers and transport planners to use the information and build applications like the TriMet Map Trip Planner [22] on top of it. Furthermore, when a GTFS feed is made publicly available on the GTFS Data Exchange website [23], the feed can be used for routing in Google Maps.

A GTFS feed consists of various files, containing detail of the movement and location of vehicles and stations operated by an agency. The minimum files required for a GTFS feed to work are: Agency, Stops, Routes, Trips, Stop Times, and Calendar.

The Agency table contains information about each transit operator. The Calendar table describes the different services that an Agency operates and specifies the time periods during which services are available. The Routes table lists all the routes operated by an Agency. Other fields in the table include the name and type of route. The Trips table defines the trips that constitute the routes in the Routes table. Routes may be linked to more than one trip. The Trips table furthermore contains information about the weekly schedule followed by the Agency as well as a direction. The direction allows agencies to distinguish between two trips that operate on the same route, but in different directions. The Stops table contains information about the locations where vehicles can be expected to stop, including a name and zone number, which can be used for calculating zone-based fares. The Stop Times table describes the sequence of stops followed on each trip in the Trip table. The arrival and departure times describe the time of arrival and departure of the vehicle at each stop on the route. The sequence of stops is described with a sequential entry for each stop. The 
Frequencies table describes the frequency with which vehicles depart from the first location on the route.

The Fare Rules table describes the conditions that need to be satisfied for a fare to be applicable on a specific route. There are different options to specify the rule. One option may be to base the fare on origin and destination zones, which will be applicable to any trips that start and end in these zones. Another option is based on a route, which only then applies to trips that are part of the specific route. Finally, it can specify a specific stop and the fare will only apply to routes containing this stop.

\subsection{OTP Workflow}

OpenTripPlanner is geared towards providing the fastest or shortest route between one or more origins and destinations. To obtain the lowest (monetary) cost route, the research necessitates the introduction of a number of modifications.

The main process of obtaining the shortest path between two points involves running two classes in OTP, namely Graph Builder and the Batch Analyst. To run Graph Builder it requires a configuration file, an OSM network, and the GTFS feed of public transport agencies to be used in the model. The Graph Builder then constructs a graph, which consists of nodes that are connected with edges. Each edge represents a path that can be followed between nodes. Edges can therefore consist of paved road segments, a specific segment on a transit route, or a section between a house and a road, which provides access from the house to the road.

The Batch Analyst then uses the graph object as input as well as a configuration file, the GTFS feeds and the origins and destinations between which routes are to be found. For each origin and destination pair, a path is found in the graph. The path consists of a series of edges, which describes the legs of the itinerary (compare the content of Fig. 5)

For the purposes of the research, the Batch Analyst in Version 0.10 .0 of OTP was adapted as follows: After the Batch Analyst finds a path in the graph, the authors iterate through the edges to distinguish between different modes of travel. For each edge, the application firstly identifies the origin node and according to its attributes, it is possible to distinguish between private vehicle legs and public transport legs. The process then distinguishes between the modes of travel to calculate cost/fares for these modes differently.

\subsubsection{Private vehicles}

According to the Automobile Association [24] the Total Operating Cost of private vehicles consists of Running Cost and Fixed Cost components. The Fixed Cost component includes vehicle finance (cost of capital), insurance, vehicle tracking and licenses. In the AA model the Fixed Cost is estimated from the purchase price and annual distance travelled. The Running Cost component includes fuel, maintenance (service and repair) and tyre wear. In

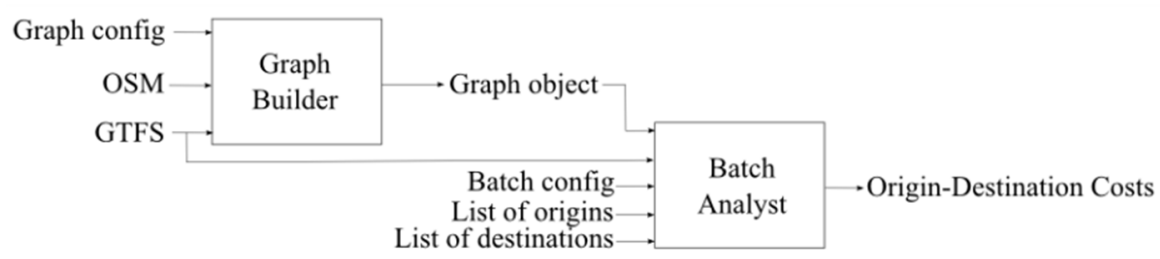

Figure 5: Process of running OTP. 
the AA model the Running Cost is estimated based on the engine capacity and current fuel price. Assuming an average vehicle purchase price of R225,000-00 (incl. VAT), average distance travelled of $27,500 \mathrm{~km}$ per annum, average engine capacity of $1600 \mathrm{~cm}^{3}$ and representative fuel price of R13.50 per litre, the Total Operating Cost per km comes to:

$\begin{array}{lll}\text { Fixed Cost } & \text { R } 2.40 & 62 \% \\ \text { Running Cost } & \text { R } 1.48 & 38 \% \\ \text { Total Operating Cost } & \text { R } 3.88 & 100 \%\end{array}$

There is a significant body of empirical knowledge on how fuel consumption varies with network and traffic conditions. In the above example the fuel cost alone represents $28 \%$ of the Total Operating Cost. The empirical results point to a simple relationship between the fuel consumption $F$ (litre $/ 100 \mathrm{~km}$ ) and the average speed of the entire trip $V$ in km per hour (total distance $D$ divided by total trip duration $T$ ).

$$
F=k_{1}+\frac{k_{2}}{V}
$$

recognising that $\mathrm{V}=\mathrm{D} / \mathrm{T}$, this can easily be converted to a fuel cost $\mathrm{C}_{\mathrm{F}}$ (Rand per km) using the trip distance $\mathrm{D}(\mathrm{km})$ and fuel price $\mathrm{P}_{\mathrm{F}}$ (Rand per litre) as follows:

$$
\begin{aligned}
& C_{F}=P_{F} F, \\
= & P_{F}\left(k_{1}+\frac{T k_{2}}{D}\right) .
\end{aligned}
$$

Due to this relationship, the fastest route found by OTP will most likely also be the lowestcost route.

\subsubsection{Transit}

For a transit-only configuration, all nodes are disregarded in the path until a node ID is found that contains the description "depart". This indicates the start of the transit trip. All subsequent edges are considered until a node is found that contains the description "arrive", which indicates the end of the transit trip. Once both the departure and arrival nodes have been found, the cost for the leg is obtained from the fares table in the agency's GTFS feed. If more transit legs are found in the path, for example due to a transfer, the fares are added to determine the total fare for the itinerary.

\section{DATA PREPARATION}

The work described in this section was taken from the validation phase of the two urban simulation projects as case studies for the research and includes the Cities of Tshwane and Nelson Mandela Bay. The objective during this phase is to create a representative transit network for the period between 2001 and 2011 which was used for validating UrbanSim and OTP as described in Subsection 7.2 hereunder. With that as objective, it was possible to use much simpler versions of the GTFS feed compared to what would be published to GTFS Exchange. Of course it would have been an advantage to start with real GTFS feeds but in South Africa only a few public transport operators use GTFS to publish their schedules. The authors consequently had no option but to set up simplified tables as described below for the City of Tshwane case study. Since only the morning peak of one weekday was used to simulate and to calculate all the measures of accessibility required by UrbanSim, many of the tables can be simplified to a fraction of the records that would normally be included. 
Various transit operators exist in the City of Tshwane, which include bus services, minibus taxis and passenger rail services. In the research model the Gautrain High Speed Rail which only started operations in 2010, was not included.

\subsection{Routes and stops}

For some of the operators, it was possible to obtain route and stop information, which were matched, merged, and then allocated the various stops to routes. From this merged file, it was possible to extract all the necessary information to generate most of the GTFS files. In the case where no stop information was available, stops were generated on the route at $1 \mathrm{~km}$ intervals. The logic behind this was that for this purpose the research was to allow a walking distance of up to $2 \mathrm{~km}$ for people in the model to access public transport. Multiple stops within the 2-km radius would therefore make no difference to the resulting accessibilities. Although minibus taxis take the liberty of stopping almost anywhere they deem necessary, the same logic as above was applied and thus include options for stops at $1 \mathrm{~km}$ intervals to ease the computational burden.

\subsection{Frequencies and travel time}

To determine the times between stops, the research assumes different average travelling speeds for each of the modes and accordingly calculated the duration. For buses, a speed of $30 \mathrm{~km} / \mathrm{h}$ was used, for minibus taxis a speed of $45 \mathrm{~km} / \mathrm{h}$, and for passenger rail a speed of $60 \mathrm{~km} / \mathrm{h}$. In the case where passenger rail, buses, and minibus taxis operate on a similar route between the same origin and destination, the passenger rail will be the most attractive since it will not only be the fastest but also the lowest cost due to the fare structure described below and in Subsection 4.3.2 above.

Since the research don't use actual schedules of the transit operators for modelling purposes, the service frequency was arbitrarily set to 60 seconds. This is clearly not representative of the real world since the research has shown that the majority of transit commuters are cost sensitive. The authors argue that in the real world commuters wait for the next service. This is to ensure that the OTP succeeds in finding a route on account of scheduling. The frequency has no influence, however, on the duration between stops; these are still dependent on the stop times that are calculated from the speed of the vehicles.

\subsection{Fares}

For passenger rail, a distance-based approach is used in which the trip cost increases with distance even though the rate per km decreases. For the research the rates given for different distance intervals and calculated a cost between each pair of stations are used. The fares ranged between R4.27 and R6.11 per trip. For bus services, a uniform trip-based fare of R8.75 per trip is used. For minibus taxis, a trip-based fare is applied. Where the methodology could not match fares with routes, a fare was allocated to the route based on the fare of a similar route. The fares ranged between R6 and R20 per trip.

\subsection{Road network}

The road network is generated from OpenStreetMap (OSM). In the research the Geofabrik $\mathrm{GmbH}$ [25] server is applied to obtain a snapshot of the OSM network for South Africa and Osmosis [26], a Java-based command-line application, to clip the network to the extent of 
the City of Tshwane. All default values of the network were kept, since the network is only used for connectivity purposes and not traffic volumes, etc.

\subsection{Origin-destination matrix/zones}

The research used the centroids of the zones described in Subsection 4.2.1 as origin and destination points and an $\mathrm{R}$ script to piece together separate output files produced by the Batch Analyst runs into one text file containing the origin, destination, OD distance on the road network and a number of monetary costs representing different mode choices aimed at specific UrbanSim sub models. These mostly include drive-alone, transit and a combined option.

\section{RESULTS}

\subsection{Accessibility}

In presenting the results of accessibilities calculated by UrbanSim from the OD matrix provided by OTP, the authors have chosen to present a measure that is much more intuitive than the logsum (eqn (1)) used by UrbanSim. Such a measure is the number of jobs that can be reached by limiting expenditure on transportation to the maximum of household affordability. This limit has rather arbitrarily been taken as $30 \%$ of household income, simply because the literature in South Africa abounds with concerns about low-income households having to spend that much on transportation.

The following two maps (Figs. 6 and 7) show the number of formal non-home-based jobs that could be reached in 2001 from any origin in the City of Tshwane without spending more than $30 \%$ of household income on transportation by different modes of transport. The origins (and destinations used by OTP) represent the zones explained in Subsection 4.2.1 above. The location of jobs was taken from the base year of the validation phase to be discussed in the next section. The first map (Fig. 6) shows the number of jobs that can be reached by private vehicle in drive-alone mode and the second map (Fig. 7) shows the number of jobs that can be reached by rail, bus or minibus taxi.

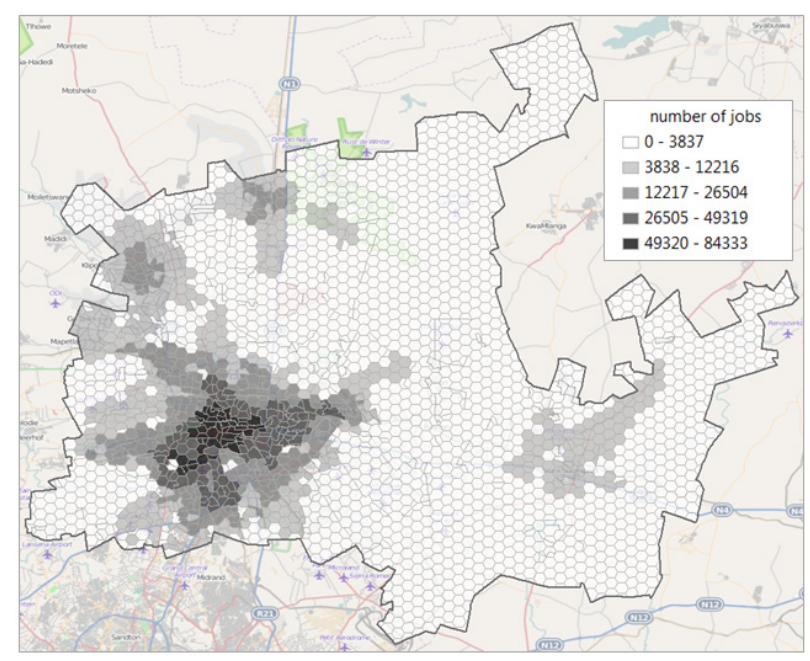

Figure 6: Number of jobs that could be reached in 2001 by spending less than $30 \%$ of household income on transportation by private vehicle. 


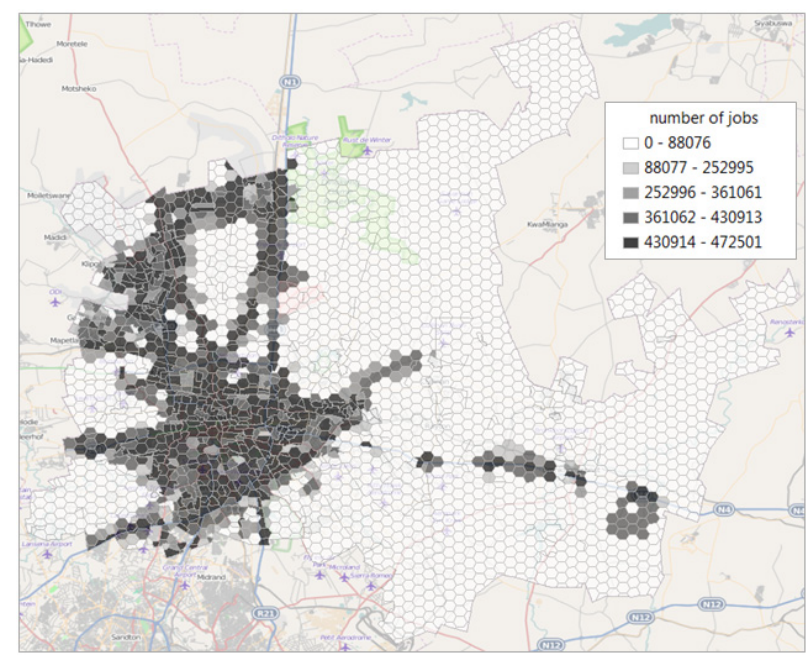

Figure 7: Number of jobs that could be reached in 2001 by spending less than $30 \%$ of household income on public transportation.

Although the maps show the enabling economic role of public transportation by providing access to jobs in many more locations, the real impact is appreciated when the number of jobs is considered that can be accessed as a percentage of the total number of jobs available in the city. By 'available', the research does not imply that the job is vacant or that a job seeker has the necessary qualifications, it simply means that if all other criteria are met, excessive transportation costs will not prevent someone from accessing an opportunity. If it is assumed that the average number of jobs that can be accessed from the 100 best placed origin zones (out of 2690) as a percentage of the total number of non-home-based jobs $(664,395)$ in 2001 the result varies from $8 \%$ with no public transport to $71 \%$ with the representative public transport used for model validation.

\subsection{Model validation}

In this section the research output illustrates the results of the preliminary validation of the Urban Simulation Model in the City of Nelson Mandela Bay case study, which is a bit closer to finality than the City of Tshwane case study model at the time of reporting on the research.

All models are validated as a matter of course by simulating a period in the past, such as the period between the 2001 and 2011 population censuses released by Statistics SA. For this purpose the model is trained on the base year data and subsequently the predictions from the simulation between 2001 and 2011 are used for validation. The authors then compare various measures of urban growth, such as the growth in number of households forecast by UrbanSim/OTP with what actually happened during the same period according to Statistics SA at a resolution of the zones described in section 4.2.1 above. Because this involves a correction to the statistics for changes in sub-place boundaries between censuses, the authors also applies a normalisation against a commercial dataset derived by Geo Terra Image (GTI) from high resolution imagery.

The results are shown in Fig. 8 as a comparison of the growth in households forecast by UrbanSim/OTP to the actual growth in households according to Statistics SA (left) as well 
as a comparison of the actual growths according to Statistics and GTI (right). Both graphs exhibit a number of serious outliers. Upon investigation of the top 20 outliers produced by UrbanSim, are all found to have logical explanations. For example a township is established in the year 2000. UrbanSim sees undeveloped prime property with a great potential return on investment and develops the area to its full potential in the next 10 years. Meanwhile in the real world there is a pending land-restitution claim on the land which prevents any development from taking place. There are various ways of informing UrbanSim about such properties, provided of course that it is known.

If the top 20 outliers are corrected, a comparison of the remaining points in Fig. 8 show that the predictive accuracy of the model is comparable to a comparison of the actual growth measured by different methods employed by two reputable organisations. The predictive accuracy of the model is estimated at about 2 housing units per hectare. While it is conceded that there is a 3-year difference in duration, the authors regard the results as highly satisfactory as an overall validation of UrbanSim and OTP used together as described in this chapter.

\section{DISCUSSION}

In the motivation for adopting measures of accessibility based on the monetary cost of commuting, which does not depend on the congested state of the network, the research departure focuses on the potential for substantial reductions in overall simulation time because it essentially replaces 5 to 30 runs of MATSim (depending on extent of austerity measures to limit interaction with UrbanSim) with a few runs of OTP (one for the base year and then only when substantial changes are introduced to the network). The reduction in simulation time for 5 to 30 runs of MATSim is not being measured in hours but in one or more days. Since OTP requires no input from UrbanSim, all the OTP work can be completed before UrbanSim starts, even on a different computer. UrbanSim has great functionality for managing scenarios and can easily be configured to calculate the accessibilities from different OTP OD matrices representing the simulation year in which significant network changes are to be introduced.
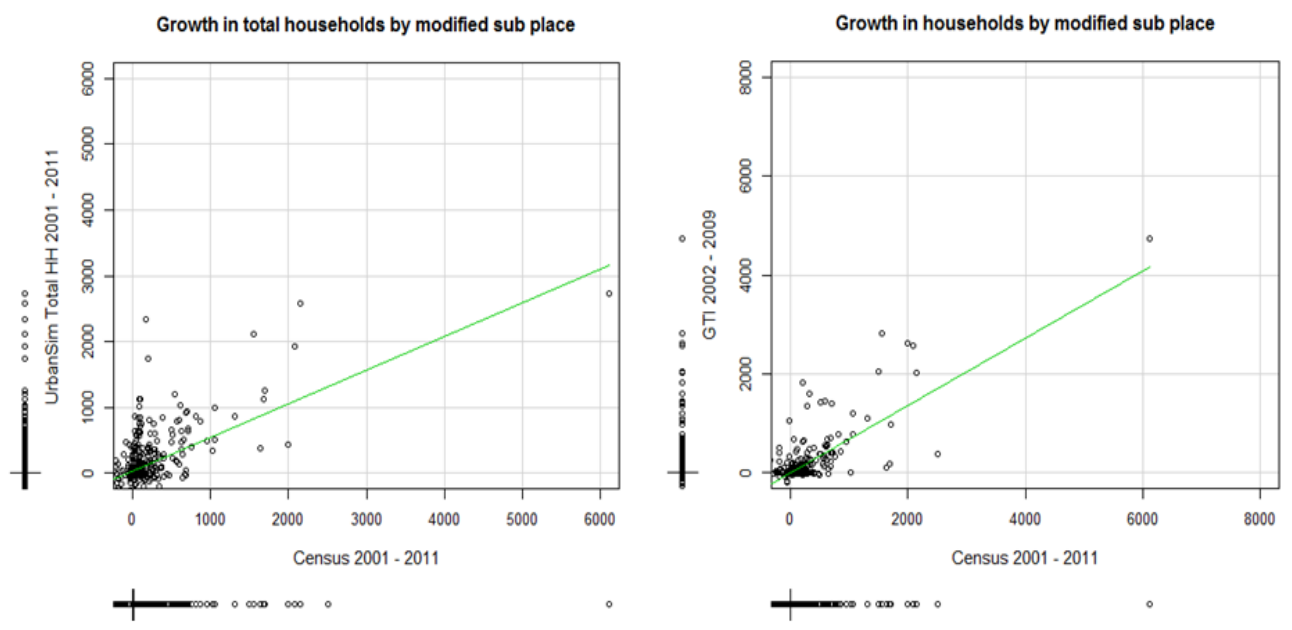

Figure 8: Model validation by comparison with actual growth according to Statistics SA (left) and actual growth according to GTI (right). 
Apart from the reduction in computing time the outcome is also superior by virtue of the fact that UrbanSim is able to calculate the accessibility measures based on the correct location of jobs for every year of the simulation. Consider for example two UrbanSim models in which one interacts with MATSim as depicted in Fig. 1 (above) and the other with OTP. In both cases the research is restricted in interaction to once every 10 years. In the first case MATSim will only be able to calculate accessibilities based on the correct location of households and jobs every 10 years and UrbanSim will have to use these for the next 9 years even though the locations are not static. This is not a limitation in the second case because OTP provides the travel cost per OD pair and UrbanSim is able to calculate accessibilities based on the correct location of jobs, also in the 9 years between interactions.

While the research has focussed on the advantages of using OTP the authors would also like to point out some disadvantages. The relative worth of the different scenarios that may be simulated for a city is usually assessed by comparing a number of indicators. If these indicators include variables such as travel time or carbon emissions from vehicles, OTP will not provide the answers because it does not model traffic volumes and congestion. In such cases the practitioner would still have to run a transportation model, but this could be restricted to one run at the start and end years of the simulation.

\section{CONCLUSION}

This chapter describes the complex nature of urban systems and examines the role of Land Use Transportation Interaction (LUTI) models in supporting the equally complex urban planning processes that shape our cities. The research supports the LUTI model that is being used to simulate spatial growth patterns in South Africa to improve understanding of the future demand for infrastructure, facilities and services inclusive of water, sanitation, electricity and transportation. This model is based on adapted versions of UrbanSim and OpenTripPlanner (OTP).

The authors present a case for using the monetary cost of commuting as an acceptable proxy for generalised cost because the majority of households in South Africa, and possibly in other developing countries, tend to be more sensitive to the cost of a trip than the duration. Since the monetary cost of commuting does not depend on the congested state of the transportation network, the research output illustrated how to modify OTP to calculate lowest-cost commuting trips by various modes of transport and provide the costs to UrbanSim for calculating measures of accessibility to jobs, which could in turn influence household location choices. In the process the research achieves substantial improvements in data preparation and simulation times. It validates the combined UrbanSim and OTP model by simulating a ten year period in the past, compares the forecasts with the actual growth in households according to two reputable sources and obtains very favourable results as output.

The research further highlights the benefits of using the General Transit Feed Specification and encourage more transit operators to follow the example of those that have already published their schedules as a means of marketing and improving their service to commuters, promoting tourism by allowing international visitors to plan itineraries before arriving in South Africa and allow more applications to be developed to inform and improve the daily experience of transit commuters.

\section{ACKNOWLEDGEMENT}

The authors gratefully acknowledge the financial support of the Department of Science and Technology (stepsa.org). 


\section{REFERENCES}

[1] Cervero, R., Linking urban transport and land use in developing countries. The Journal of Transport and Land Use, 6(1), pp. 7-24, 2013.

[2] United Nations, The millennium development goals report 2011. Technical report, United Nations, New York, 2011, http://www.un.org/millenniumgoals/reports.shtml

[3] Statistics South Africa, Census 2011, http://www.statssa.gov.za/census2011/ default.asp

[4] Wegener, M., Overview of land-use transport models. Transport Geography and Spatial Systems. Handbook 5 of the Handbook in Transport, eds D.A. Hensher and K. Button, Pergamon/Elsevier Science: Kidlington, UK, pp. 127-146, 2004.

[5] Wegener, M., Simulation of land use and transport: State of the art. SustainCity Conference, ETH Zurich, 17-18 April 2013.

[6] Waddell, P., Confronting the bane of endogeneity in modelling urban social dynamics. Workshop on Modelling Urban Social Dynamics, University of Surrey, 2005.

[7] Wegener, M., Operational urban models: State of the art. Journal of the American Planning Association, 60(1), pp. 17-29, 1994.

[8] Simmonds, D., Waddell, P. \& Wegener, M., Equilibrium v. dynamics in urban modelling. Paper presented at the Symposium on Applied Urban Modelling (AUM 2011) "Innovation in Urban Modelling" at the University of Cambridge, 23-24 May 2011.

[9] Waddell, P. \& Ulfarsson, G.F., Introduction to urban simulation: Design and development of operational models. Handbook in Transport, Volume 5: Transport Geography and Spatial Systems, eds Stopher, Button, Kingsley \& Hensher, Pergamon Press, pp. 203-236, 2004.

[10] McFadden, D., Conditional logit analysis of qualitative choice behavior. Frontiers in Econometrics, ed. P. Zarembka, Academic Press: New York, pp. 105-142, 1974.

[11] McFadden, D., Econometric models of probabilistic choice. StrucAppendix A: Data Preparation in a Sparse Data Environment, eds C. Manski \& D. McFadden, 1981.

[12] Nicolai, T.W. \& Nagel, K., Investigating accessibility indicators for feedback from a travel to a land use model. SustainCity Working Paper, 6.2, TU Berlin, 2011.

[13] Awaludin, A. \& Chen, D., UrbanSim Parallel Programming Capstone Paper University of Washington, Computer Science and Engineering 481E, June 4, 2007.

[14] Raney, B. \& Nagel, K., Truly agent-based strategy selection for transportation simulations, 2002, http://www.ltrc.lsu.edu/TRB 82/TRB2003-002258.pdf

[15] Zöllig Renner, C., Schirmer, C.P. \& Müller, K., Case Study Zurich Simulation, Zurich, April 2013.

[16] Hansen, W., How accessibility shapes land use. Journal of the American Planning Association, 25(2), pp. 73-76, 1959.

[17] Moeckel, R., Business location decisions and urban sprawl - a microsimulation of business relocation and firmography, $\mathrm{PhD}$ thesis, Department of Spatial Planning at the University of Dortmund, 2006.

[18] Visagie, J., Who are the middle class in South Africa? Does it matter for policy? 2013, www.econ3x3.org/article/who-are-middle-class-south-africa-does-it-matterpolicy\#sthash.1XzwvRCb.dpuf

[19] Visagie, J. \& Posel, D., A reconsideration of what and who is middle class in South Africa. ESSA Conference, University of Kwazulu-Natal, 2011.

[20] National Household Travel Survey (NHTS), Statistical Release PO320, 2013.

[21] Google Developers, The General Transit Feed Specification Reference, https://developers.google.com/transit/gtfs/reference 
68 Transportation, Land Use and Integration: Perspectives for Developing Countries

[22] TriMet 2015, Map Trip Planner, http://trimet.org/howtoride/maptripplanner.htm

[23] GTFS Data Exchange, http://www.gtfs-data-exchange.com/

[24] Automobile Association, http://www.aa.co.za/on-the-road/calculator-tools/ratescalculator.html

[25] Geofabrik GmbH, http://download.geofabrik.de/africa/south-africa-and-lesotho.html

[26] Osmosis 2015, http://wiki.openstreetmap.org/wiki/Osmosis 